\title{
Vapour sorption and permeation properties of poly (dimethylsiloxane) films*
}

\author{
I. Blume, P.J.F. Schwering, M.H.V. Mulder and C.A. Smolders** \\ University of Twente, Department of Chemical Technology, P.O. Box 217, 7500 AE Enschede \\ (Netherlands)
}

(Received October 29, 1989)

\begin{abstract}
Sorption and permeation of several organic vapours in poly (dimethylsiloxane) were investigated. Solubility and permeability coefficients measured show a strong dependence of the applied vapour activity typical for these systems, contrary to the behaviour of permanent gases. However, as irregularities in the series of chloromethanes show, highest sorption does not necessarily lead to the highest permeability value. A possible explanation of this effect can be found in the concentration dependent diffusion behaviour of poly (dimethylsiloxane) toward these organic vapours. Thus, from the kinetics of solvent uptake, i.e. the sorption isotherms over time, the diffusion coefficients, which were corrected for swelling and thermodynamic activity of the vapour, were calculated for the different vapour activities.
\end{abstract}

Keywords: poly (dimethylsiloxane); sorption and permeation; chloromethanes

\section{Introduction}

During the last years the transport behaviour of organic vapours through polymeric membranes has again gained further interest. Particularly during the forties till sixties of this century, the study on diffusion and permeation of organic compounds in poly (dimethylsiloxane) was in a strong upswing; regrettingly the research efforts decreased in the years thereafter. Recently, research has spurred more interest in these areas partly due to upcoming practical applications using newer methods of membrane separation. Here may be mentioned the recovery or recycling of organic vapours from air streams and the separation of aqueous-organic or organic-organic mixtures via pervaporation.

In dense polymer films, where the solution-diffusion mechanism holds, the

\footnotetext{
*Paper presented at the 6th International Symposium on "Synthetic Membranes in Science and Industry", Tübingen, September 4-8, 1989.

**To whom correspondence should be addressed.
} 
transport of organic vapours can be investigated by measurements of permeation, sorption and diffusion. For the case of organic vapour permeation, it can be seen that concentration dependent sorption has a major influence on the overall transport characteristics of the membrane and leads to extremely high vapour permeability coefficients. A more fundamental understanding of the sorption and permeation effects will help to gain further insight into the transport of organic vapours through rubbery polymers. Many studies on diffusion, and also sorption and permeation, of organic components in various rubbers can be found in a vast number of papers. A review of Rogers back in 1965 already cited some odd 350 references [1]. Many excellent papers and reviews on this subject can be found and the reader is referred to these for further information [2-10]. A lot of studies, however, sometimes lack certain important points. Either the range of penetrant concentration covered was rather small or the studies only included either sorption or diffusion. It seems nevertheless necessary, especially if one likes to obtain a more complete picture on the transport, to do a more complete study, i.e. measure both permeation and sorption over a whole range of activities, from which then the diffusion behaviour can be deduced.

The goal of this study was to examine a large group of different organic solvents in their permeation and sorption behaviour through poly(dimethylsiloxane), or silicone rubber as it is commonly called. In particular, this paper will concentrate on the three solvents methylene chloride, chloroform and carbontetrachloride, which are part of the homologous series of the chloromethanes, and we will describe permeation and sorption experiments that were done over a larger range of solvent concentrations or activities.

\section{Experimental}

\section{Membranes}

Poly (dimethylsiloxane) was chosen as polymer material mainly for two reasons. Firstly, this polymer has shown extremely good permeation rates together with reasonable selectivities, and secondly it has been under study in the past, which allows for data comparison. For this study, films of varying thickness were prepared from a two-component rubber, RTV 615 (General Electric), which is crosslinked via an addition hydrosililation reaction. Films were prepared by a standard casting technique on plexiglas plates and crosslinked at $80^{\circ} \mathrm{C}$ for $15 \mathrm{hr}$ to yield clear and transparent membranes.

\section{Solvents}

As model solvents a group of 14 organics was selected from different chemical groups, i.e. alcohol, ketone, ester, aliphatic and aromatic hydrocarbon, and a series of chlorinated hydrocarbons on the basis of their industrial relevance. All the solvents were of analytical grade and used without further purification. 


\section{Apparatus}

Using a continuous gravimetric method, the kinetics of mass uptake and the equilibrium sorption behaviour of thick poly (dimethylsiloxane) films has been studied for a variety of organic solvents at different vapour pressures. The apparatus, shown in Fig. 1, mainly consists of two temperature regions, i.e. constant temperature baths, and an electrical balance. Solvent vapour is generated at a constant temperature $T_{1}$ [vapour pressure $p_{1}=f\left(T_{1}\right)$ ]. An air stream saturated with this vapour is blown over the polymer sample, which is suspended on an electronic balance in a second temperature bath $\left(T_{2}>T_{1}\right)$. Several coil heat-exchangers are included to secure temperature equilibrium of the air stream. Mass uptake until equilibrium is continuously monitored on an electrical chart recorder.

Besides the vapour sorption studies, static and dynamic sorption experiments from the liquid phase were performed. Polymer samples were submerged in bottles containing liquid solvent, which were placed in a constant temperature bath. Mass uptake was monitored either by periodically removing, drying by paper blotting and weighing a single polymer sample or by using several

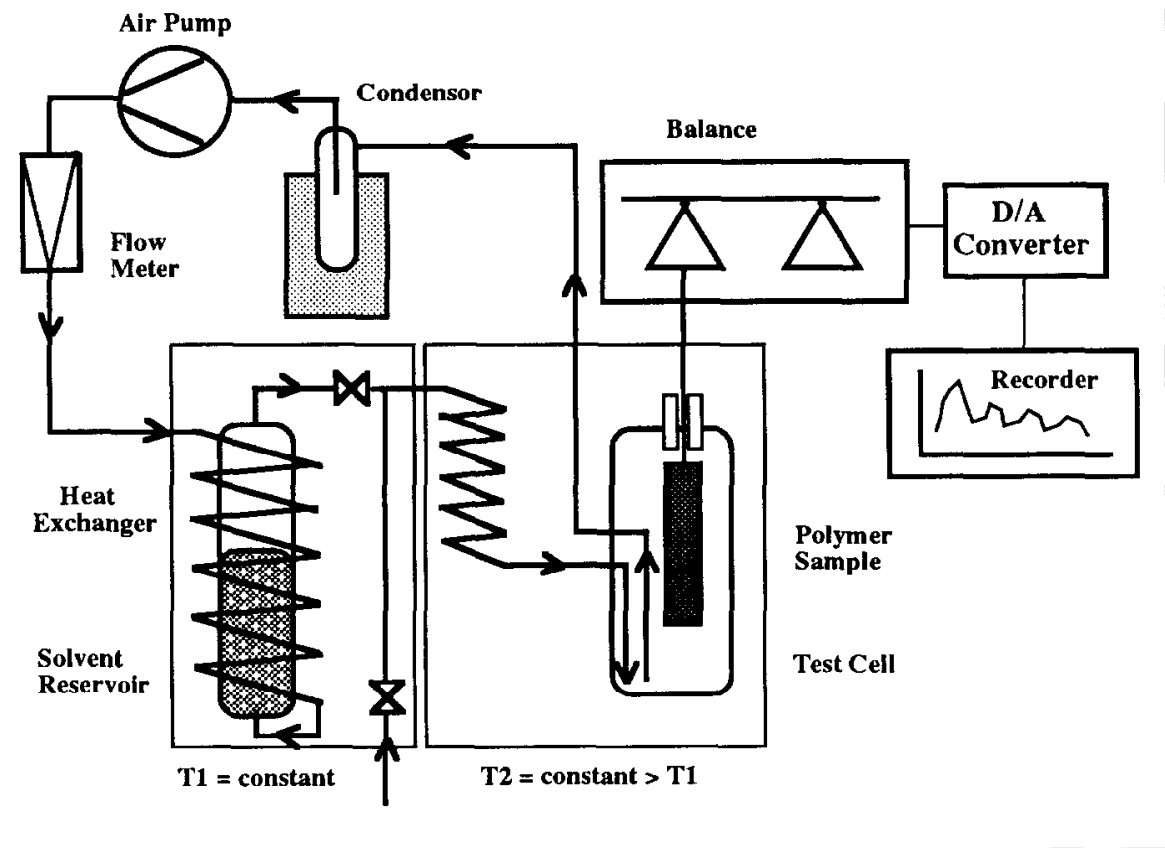

Fig. 1. Vapour sorption apparatus (explanation see text). 


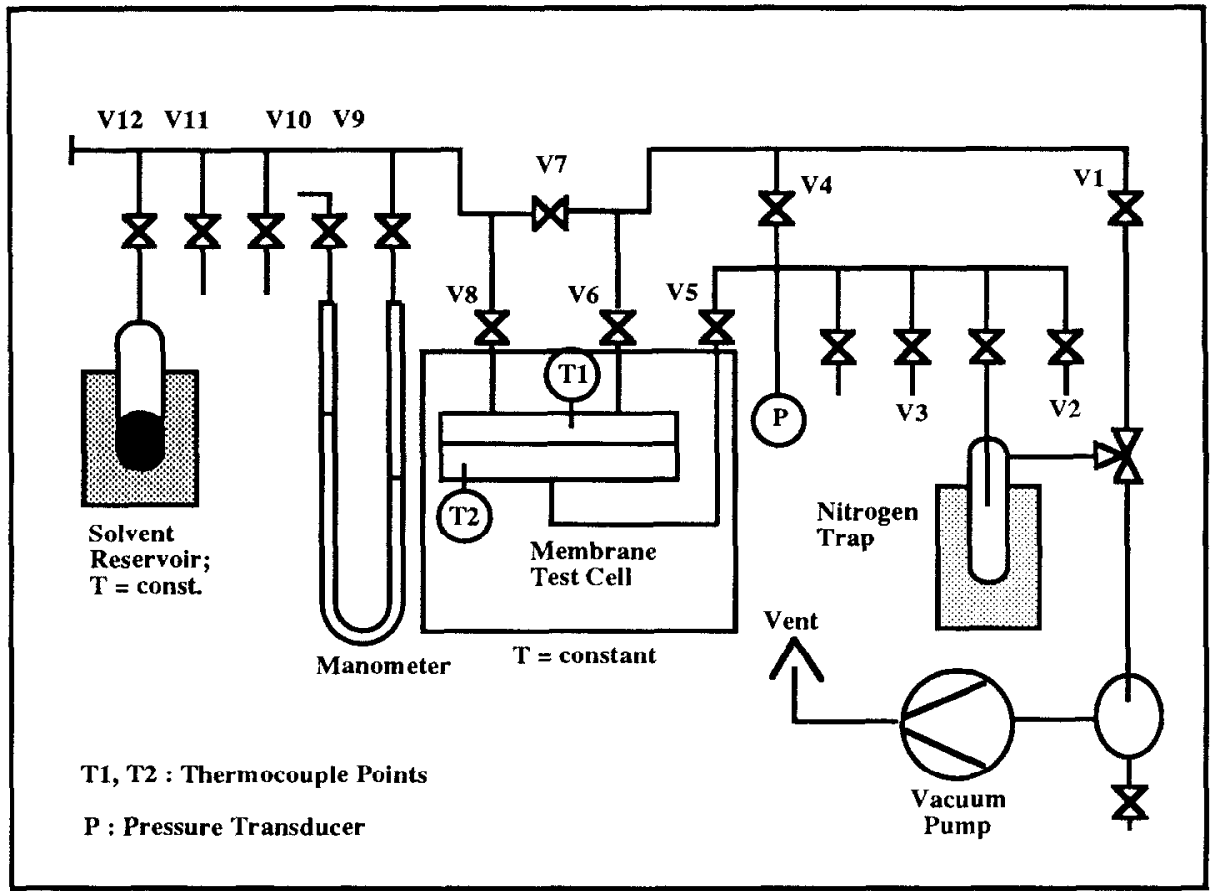

Fig. 2. Vapour permeation apparatus (explanation see text).

samples simultaneously and weighing each after an appropriate time interval. Equilibrium sorption was determined after $24 \mathrm{hr}$ and one week of submersion. No significant difference could be observed, indicating equilibrium was obtained after $24 \mathrm{hr}$.

Permeation experiments for these solvents were performed on a typical vacuum test apparatus (Fig. 2). The solvent vapour generated by a constant temperature bath $\left(G_{1}\right)$ permeates through the membrane (which is at a higher temperature $T_{2}>T_{1}$ ) because of a very low and constant downstream pressure. here the permeate is constantly condensed with liquid nitrogen. Fluxes are calculated from the weight over time measurements using appropriate relationships. Different vapour pressures can be obtained by changing the temperature $T_{1}$. Liquid permeation was measured by using a liquid feed reservoir above the membrane test cell.

\section{Results}

\section{Sorption and permeation from the liquid phase}

Liquid sorption and permeation of most of the selected organics were performed to obtain a first overview of their transport behaviour. Table 1 shows the results, which were measured at a temperature of $40^{\circ} \mathrm{C}$. For comparison, 
TABLE 1

Liquid permeability and sorption of organic solvents through silicone rubber

\begin{tabular}{lcc} 
& Permeability (barrer) & Sorption $(\mathrm{g} / 100 \mathrm{~g})$ \\
\hline Nitrogen & 280 & $0.011 @$ latm \\
Oxygen & 600 & 0.026 \\
Methane & 940 & 0.034 \\
Carbon dioxide & 3,200 & 0.282 \\
Water & 23,000 & 0.38 \\
Ethanol & 45,000 & 6.5 \\
Methylene chloride & 165,000 & 154 \\
Chloroform & 283,000 & 207 \\
Carbontetrachloride & 180,000 & 241 \\
1.2-Dichloroethane & 248,000 & 58 \\
1.1.1-Trichloroethane & 247,000 & 193 \\
Trichloroethylene & 614,000 & 235 \\
Toluene & $1,460,000$ & 115 \\
\hline
\end{tabular}

Temperature: $40^{\circ} \mathrm{C} ; 1$ barrer $=1 \times 10^{-10} \mathrm{~cm}^{3}(\mathrm{STP})-\mathrm{cm} /\left(\mathrm{cm}^{2}-\mathrm{sec}-\mathrm{cmHg}\right)$.

values for some permanent gases obtained from the literature [11] are included too.

Remarkable differences can be seen from this table. The permeability covers four orders of magnitude, e.g. the ratio of toluene to nitrogen is about 5200 . Similar differences can be found in sorption, the ratio of carbontetrachloride toward water is about 630 . The sorption of the permanent gases is even lower. Another peculiarity can be seen among the group of chloromethanes, i.e. methylene chloride, chloroform and carbontetrachloride. Sorption increases with increasing number of chlorine atoms or molar volume, as it would be predicted from simple solubility parameter considerations. Permeability, however, shows a distinct maximum for chloroform, which is outside of the error margins of the experiments. The transport behaviour of this group of chloromethanes was therefore studied more closely, i.e. sorption and permeation experiments were done over a larger range of vapour activities.

\section{Sorption from the vapour phase}

Typical mass uptake over time curves for the three solvents methylene chloride, chloroform and carbontetrachloride for various vapour activities were obtained. Sorption occurs very fast where $80 \%$ of the equilibrium value is already reached after only a few minutes. The sorption isotherms of poly(dimethylsiloxane) at $40^{\circ} \mathrm{C}$, which were calculated from these data, are shown in Fig. 3. The progressive increase of the solubility with increasing vapour pressure can nicely be seen (for permanent gases, sorption $c$ over pressure $p$ would yield a straight line according to Henry's law). These sorption isotherms 


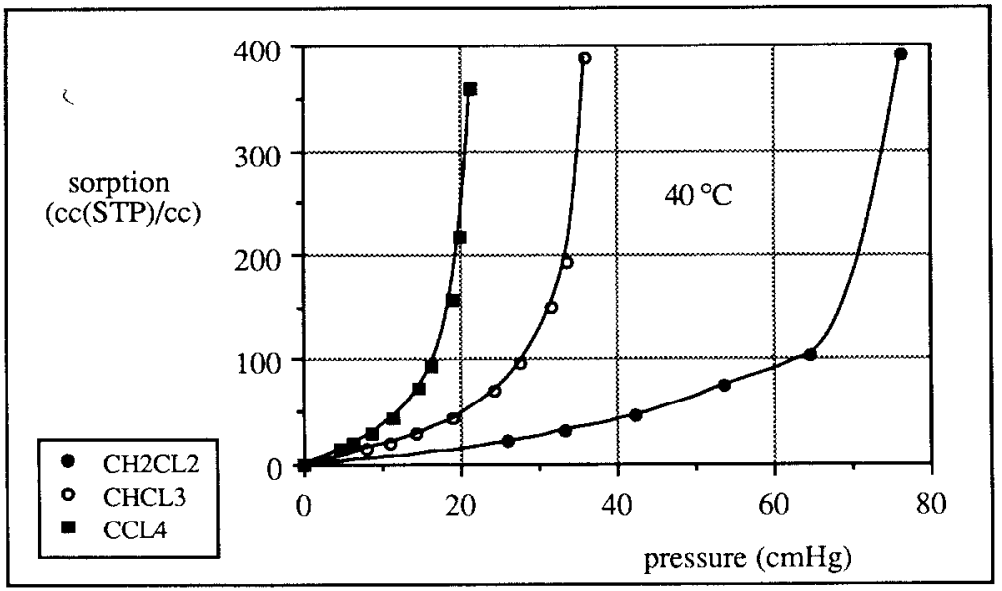

Fig. 3. Sorption isotherms of three chloromethanes in poly (dimethylsiloxane) at $40^{\circ} \mathrm{C}$.

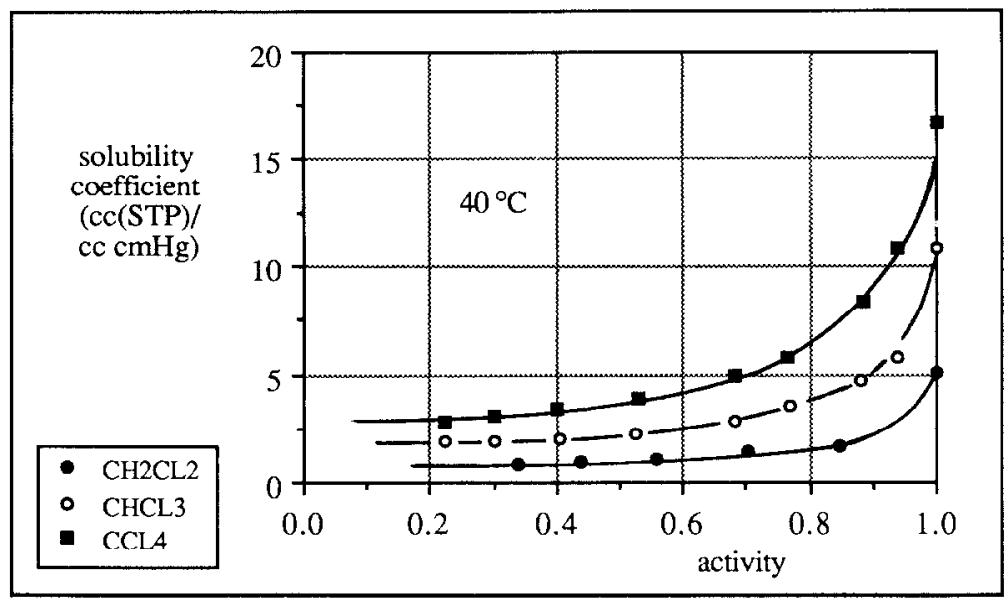

Fig. 4. Solubility coefficients of three chloromethanes in poly (dimethylsiloxane) at $40^{\circ} \mathrm{C}$.

show a strong non-ideal behaviour, as the plot of the solubility coefficients $S(c)=c / p\left(\mathrm{~cm}^{3}\right.$ (STP $\left.) / \mathrm{cm}^{3}-\mathrm{cmHg}\right)$ in Fig. 4 also indicates. At low penetrant activity, a constant Henry-like solubility coefficient is found, which progressively increases with higher activity. The tendency of increasing sorption going from methylene chloride to carbontetrachloride is again found back over the whole range of vapour activity.

\section{Permeation from the vapour phase}

Permeability measurements of the three chloromethanes are shown in Fig. 5. Unexpectedly, the permeability shows a maximum for chloroform, which is true for all vapour activities measured. From the sorption data seen above, 


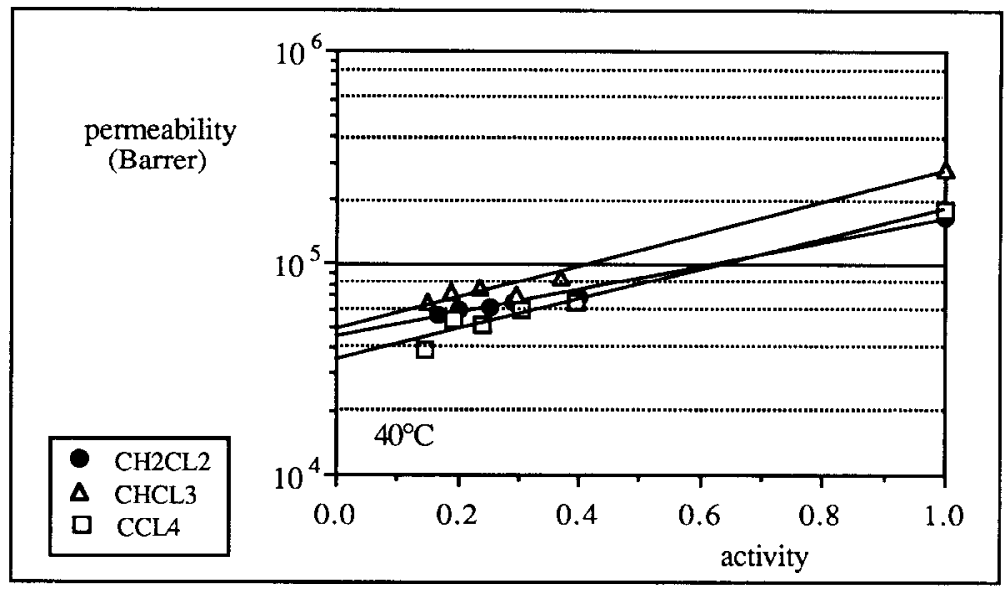

Fig. 5. Permeability of three chloromethanes through poly (dimethylsiloxane) at $40^{\circ} \mathrm{C}$.

which were obtained from both liquid and vapour phase, a steady increase in solvent uptake with increasing chlorine content was found. The question is what causes this shift in transport behaviour. Obviously, the maximum in permeability for chloroform can only be explained realizing that the diffusion of the larger carbontetrachloride has a retarding influence, leading to a lower overall permeability compared with chloroform.

\section{Diffusion behaviour}

As mentioned above, an answer for the maximum in permeability might be found in the diffusion behaviour of these solvents. The kinetic sorption data were recalculated, and from the linear parts of the normalized sorption isotherms, in which $M_{\mathrm{t}} / M_{\infty}$ is plotted at various activities as a function of thickness-reduced time, mean integral diffusion coefficients were calculated following the analysis of Crank [12], showing a strong concentration dependence, i.e. a decrease with activity or solvent volume fraction. These integral diffusion coefficients are very complex functions, representing mean values over the concentration range used for the appropriate sorption time curve. Crank and Henry [13] found that the following equation is applicable, which relates $D$ to the differential diffusion coefficient $\bar{D}(c)$ :

$\bar{D}=\frac{1}{c_{\mathrm{o}}} \int D(c) \mathrm{d} c \quad$ or $\frac{\mathrm{d}\left(\bar{D}^{*} c\right)}{\mathrm{d} c}=D(c)$

This diffusion coefficient normally starts out at low values at lower activities, increases first and then begins to decrease at higher solvent activities, i.e. one often finds a maximum at some solvent concentration. However, this effect is not unusual and can be found back in literature (see e.g. [14-16]). The 

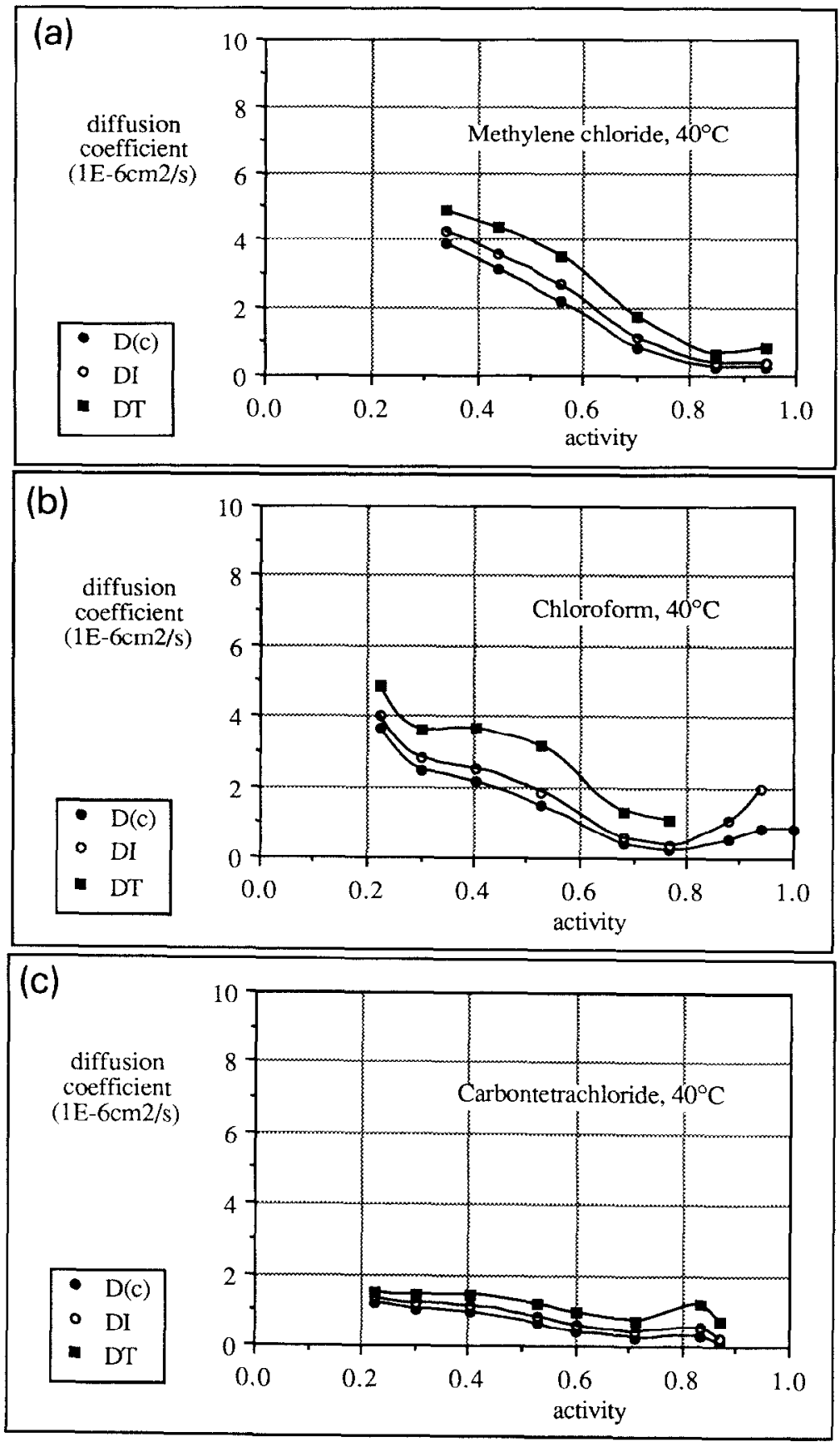

Fig. 6. Diffusion coefficients $D(c), D_{\mathrm{I}}$ and $D_{\mathrm{T}}$ vs. activity of methylene chloride (a), chloroform (b), and carbontetrachloride (c) in poly (dimethylsiloxane) at $40^{\circ} \mathrm{C}$. 
reason for this seems to be the enormous swelling of the polymer (i.e. the assumed frame of reference of constant polymer dimensions is no longer true, one must transform to a frame of constant polymer dimensions and a plane of zero mass flow ). One then arrives at the intrinsic diffusion coefficient $D_{\mathrm{I}}$, which is a measure of solvent mobility in the polymer, defined by:

$D_{\mathrm{I}}=D /\left(1-\Phi_{\mathrm{s}}\right)^{5 / 3}$

where $D$ is the mean diffusion coefficient obtained from the sorption experiment and $\Phi_{\mathrm{s}}$ is the penetrant volume fraction.

The thickness correction factor $5 / 3$ in eqn. (2) arises from the fact that sorption was measured on a freely suspended polymer sample, i.e. for isotropic swelling of the sample. Usually, for glassy polymers a factor of $9 / 3$ can be found, which is valid for one-dimensional swelling in the direction of the thickness and with no lateral swelling [15].

To prove that isotropic swelling is a valid assumption for silicone rubber, liquid sorption, which shows the largest effects, was measured via two different methods. The weight increase over time was compared to experiments where the area increase of the film sample was measured. Assuming isotropic swelling behavior of the polymer, a change in area can be related to volume and hence to the weight increase. Taking also the root of the area, the increase of thickness can be determined. This method seems only suitable for polymers with relatively large swelling. Nevertheless, as a result it was found that the two methods agreed within experimental error with each other, and thus silicone rubber swells isotropically.

Another factor for the strong concentration dependence is that one also has to correct for the thermodynamic activity of the solvent vapour, i.e. correct with $\partial \ln a / \partial \ln \Phi$ (which is the derivative of the activity coefficient) $[4,11,15,17]$. This function can be obtained from any suitable expression for the sorption isotherm, e.g. the Flory-Huggins equation [16]. Equation (2) than expands to:

$D_{\mathrm{T}}=D /\left[\left(1-\Phi_{\mathrm{S}}\right)^{5 / 3} \partial \ln a / \partial \ln \Phi_{\mathrm{s}}\right]$

As a first approximation, the simple Flory-Huggins equation with a constant interaction parameter was used, which is a prerequisite to be able to differentiate $\partial \ln a / \partial \ln \Phi_{\mathrm{S}}$.

Looking now at the three chloromethanes (Fig. 6) $D(c), D_{\mathrm{I}}$ and $D_{\mathrm{T}}$ show a decrease going from methylene chloride to carbontetrachloride, which can explain the decreased permeability of the latter solvent with regard to chloroform. The diffusion coefficients $D_{\mathrm{T}}$ show much less of a concentration dependence, however, are still not linear.

\section{Discussion}

In the previous section, sorption and permeation data were presented, from 

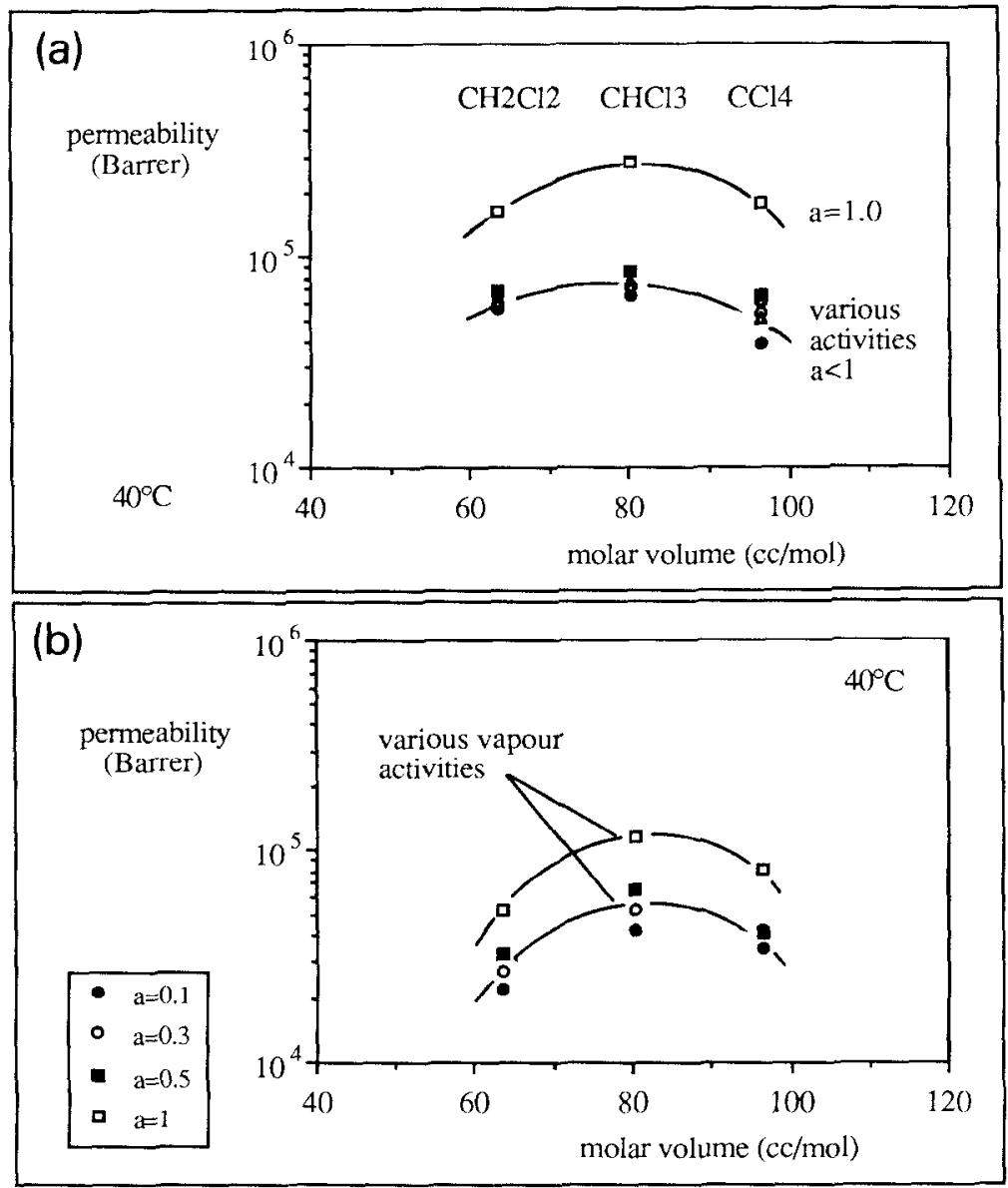

Fig. 7. Permeability coefficients $P$ (from steady-state permeation) (a) and $P_{\mathrm{s}}$ (from sorption kinetics) (b) vs. solvent molar volume of three chloromethanes.

which the concentration dependent diffusion coefficients were derived. Replotting the data, it seems possible to explain the rather strange maximum of the chloroform permeability if one combines both solubility and diffusivity.

The diffusion coefficients $D_{\mathrm{T}}$ were taken to calculate a permeability from sorption analysis as a function of the vapour activity via:

$P_{\mathrm{S}}(c)=D_{\mathrm{T}}(c) S(c)$

with $S(c)$ the solubility coefficient (see Fig. 5 ).

It should be stressed that it is not our intention to derive a generalized possibility to calculate permeabilities from sorption and diffusion experiments. After all, permeabilities can be measured directly in a relatively easy way. The calculation is done only as we believe that it helps to explain the strange be- 


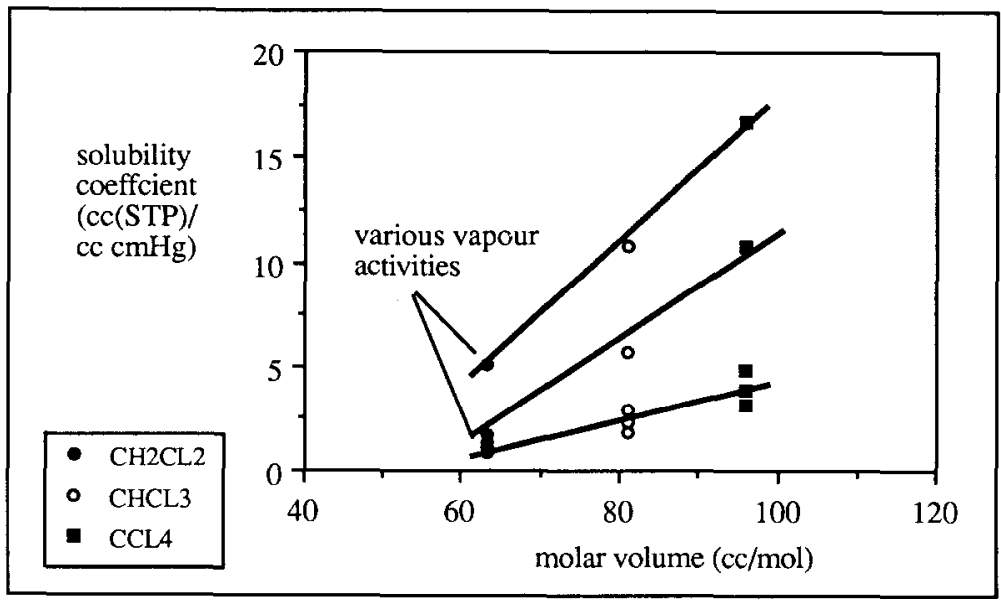

Fig. 8. Solubility coefficients vs. solvent molar volume of three chloromethanes in poly (dimethylsiloxane) at $40^{\circ} \mathrm{C}$.

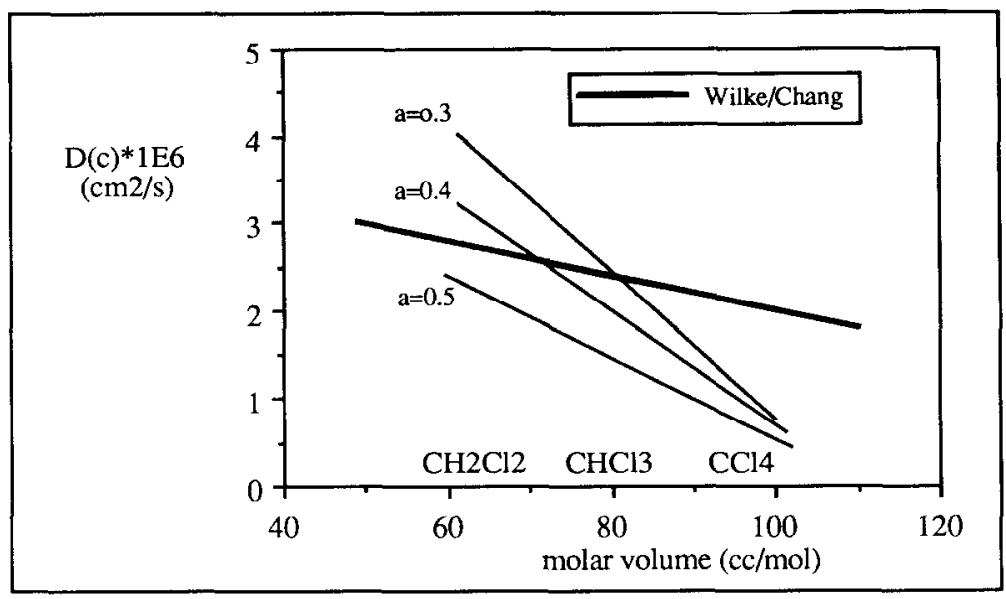

Fig. 9. Diffusion coefficients vs. solvent molar volume of three chloromethanes in poly (dimethylsiloxane) at $40^{\circ} \mathrm{C}$. The straight line is calculated from an empirical modification of the Einstein relation after Wilke and Chang.

haviour found for the chloromethanes. Figure 7 shows permeability coefficients obtained from experiments and calculated from eqn. (4). As seen, at all activities shown, the maximum in the chloroform permeability is found back. Which is an indication that it is the diffusion of the three penetrants that causes this effect.

The solubility coefficients of the three chloromethanes are shown in Fig. 8 as a function of their molar volume, which is a measure of the solvent size. Obviously, the solubility increases with both activity and molar volume. This 
fact is commonly known because solubility increases with the boiling or critical point of the penetrants, or to say it in other words, solubility increases with the condensibility of the solvent $[6,19]$.

The differential diffusion coefficient $D(c)$, which is plotted versus molar volume (Fig. 9), shows a different behaviour. As expected, $D(c)$ decreases with increased molar volume. However, the decrease for carbontetrachloride is more than linear if compared with the two other solvents, indicating a stronger interaction with the polymer or a stronger steric hindrance. For comparison, the diffusion coefficient of these three penetrants was calculated using an empirical modification of the Einstein relation of Wilke and Chang [15,20]. The underlying thought being that the highly swollen silicone rubber has a somewhat liquid-like nature. This simple equation however completely underestimates the effect of solvent size for diffusion in the polymer, showing a much smaller size dependence. It is now the fine interplay of solubility and diffusivity that gives the surprising effect of a permeability maximum.

\section{Conclusions}

Using silicone rubber a study on the sorption and permeation behaviour of various organic solvents in dependence of vapour concentration was performed. Permeabilities of solvents are strongly dependent on applied vapour pressure, i.e. concentration. The permeation is mainly determined by the large sorption of solvent into the polymer. Solubilities of the solvents in the polymers show a strong non-linear behaviour, clearly indicating again a concentration dependent relationship, $S(c)$.

In the analysis of the permeation and sorption behaviour of three chloromethanes, methylene chloride, chloroform and carbontetrachloride, concentration dependent diffusion coefficients were calculated from sorption kinetics to explain the rather curious result of a maximum in chloroform permeability.

\section{Acknowledgements}

The authors gratefully acknowledge the support of this work provided by a grant of the Dutch Ministry of Economic Affairs under their IOP-M program and the financial support of TNO, AKZO Zout Corp. and Shell Corp.

\section{References}

1 C.E. Rogers, Solubility and diffusivity, in: D. Fox, M.M. Labes and A. Weissberger (Eds.), Physics and Chemistry of the Organic Solid State, Vol. 2, Wiley-Interscience, New York, NY, 1965, Chap. 6.

2 R.M. Barrer, Diffusion in an through Solids, Cambridge University Press, Cambridge, 1951.

3 R.M. Barrer and G. Skirrow, Transport and equilibrium phenomena in gas-elastomer systems, J. Polym. Sci., 3(4) (1948) 549-563 and 564-575.

4 R.M. Barrer and R.R. Fergusson, Diffusion of benzene in rubber and polythene, Trans. Faralay Soc., 54 (1958) 989-1000. 
5 V. Stannett, M. Szwarc, R.L. Bhargava, J.A. Meyer, A.W. Myers and C.E. Rogers, Permeability of Plastic Films and Coated Paper to Gases and Vapors, TAPPI Monograph Ser. No. 23, published by TAPPI, New York, NY, 1962.

6 G.J. van Amerongen, Diffusion in elastomers, Rubber Chem. Technol., 37 (1964) 1065-1152.

7 R.M. Barrer and H.T. Chio, Solution and diffusion of gases and vapors in silicone rubber membranes, J. Polym. Sci., C10 (1965) 111-138.

8 J. Crank and G.S. Park (Eds.), Diffusion in Polymers, Academic Press, London and New York, 1968.

9 M.S. Suwandi and S.A. Stern, Transport of heavy organic vapors through silicone rubber, J. Polym. Sci., Polym. Phys. Ed., 11 (1973) 663-681.

10 W.J. Koros and R.T. Chern, Separation of gaseous mixtures using polymer membranes, in: R.W. Rousseau (Ed.), Handbook of Separation Process Technology, Wiley-Interscience, New York, NY, 1987, Chap. 20.

11 S.A. Stern, V.M. Shah and B.J. Hardy, Structure-permeability relationships of silicone polymers, J. Polym. Sci., Polym. Phys. Ed., 25 (1987) 1263-1298.

12 J. Crank, The Mathematics of Diffusion, 2nd edn., Clarendon Press, Oxford, 1975.

13 J. Crank and M.E. Henry, Diffusion in media with variable properties, Trans. Faraday Soc., 45 (1949) 636-650.

14 P. Meares, Polymers-Structure and Bulk Properties, Van Nostrand, London and New York, 1965.

15 G.S. Park, Transport in polymer films, in: R.R. Myers and J.S. Long (Eds.), Characterization of Coatings: Physical Techniques, Vol. 2, Part II, Marcel Dekker, New York, Chap. 9, NY, 1976.

16 J. Hauser, A. Heintz, G.A. Reinhardt, B. Schmittecker, M. Wesslein and R.N. Lichtenthaler, Sorption, diffusion and pervaporation of water/alcohol mixtures in PVA-membranes. Experimental results and theoretical treatment, Proc. 2nd Int'l Conf. on Pervaporation Processes in the Chemical Industry, R. Bakish (Ed.), BMC, New Jersey, 1987, 15-34.

17 M. Fels and R.Y.M. Huang, Diffusion coefficients of liquids in polymer membranes by a desorption method, J. Appl. Polym. Sci., 14 (1970) 523-536.

18 P.J. Flory, Principles of Polymer Chemistry, Cornell University Press, Ithaca, NY, 1953.

19 R.W. Baker and I. Blume, Permselective membranes separate gases, CHEMTECH, 16(4) (1986) 232-238.

20 R.C. Reid, J.M. Prausnitz and B.E. Poling, The Properties of Gases and Liquids, 4th edn., McGraw-Hill, New York, NY, 1987. 